\title{
Finite Groups in which $\tau$-quasinormality is a Transitive Relation
}

\author{
Vladimir O. Lukyanenko - Alexander N. Skiba
}

ABSTRACT - Let $H$ be a subgroup of a finite group $G$. We say that $H$ is $\tau$-quasinormal in $G$ if $H P=P H$ for all Sylow $p$-subgroups $P$ of $G$ such that $(|H|, p)=1$ and $\left(|H|,\left|P^{G}\right|\right) \neq 1$. In this article, finite groups in which $\tau$-quasinormality is a transitive relation are described.

\section{Introduction.}

Throughout this paper, all groups are finite.

An interesting question in finite group theory is to determine the influence of the embedding properties of members of some distinguished families of subgroups on the structure of the group. The present paper adds some results to this line of research.

Let $H$ be a subgroup of a group $G$. Then $\pi(G)$ denotes the set of all primes dividing $|G| ; H^{G}$ is the normal closure of $H$ in $G$, that is, the intersection of all normal subgroups of $G$ containing $H ; H_{G}$ is the normal core of $H$ in $G$, that is, the product of all normal subgroups of $G$ contained in $H$. Recall that a subgroup $H$ of a group $G$ is said to be $S$-permutable, $S$-quasinormal, or $\pi(G)$-permutable in $G$ (Kegel [22]) if $H P=P H$ for all Sylow subgroups $P$ of $G$; the subgroup $H$ is said to be $S$-semipermutable, $S$ semiquasinormal, or $S$-seminormal in $G$ if $H P=P H$ for all Sylow $p$-subgroups $P$ of $G$ with $(|H|, p)=1$.

We shall use the notion of $\tau$-quasinormal embedding introduced in [28]: a subgroup $H$ of a group $G$ is said to be $\tau$-quasinormal in $G$ if $H P=P H$ for all Sylow $p$-subgroups $P$ of $G$ such that $(|H|, p)=1$ and

Indirizzo degli A.: Department of Mathematics, Gomel Francisk Skorina State University, Gomel 246019, Belarus.

E-mail: vladimir.lukyanenko84@gmail.com alexander.skiba49@gmail.com 2000 Mathematics Subject Classification: 20D10, 20 D15. 
$\left(|H|,\left|P^{G}\right|\right) \neq 1$. It is clear that every $S$-semiquasinormal subgroup is $\tau$-quasinormal. The following example shows that in general the set of $\tau$-quasinormal subgroups is wider than the set of all $S$-semiquasinormal subgroups and, of course, than the set of all $S$-quasinormal subgroups.

EXAMPLE 1. Let $p<r<q$ be primes, $R$ any cyclic $r$-group and $Q$ a faithful irreducible $R$-module over $\mathrm{F}_{q}$. Let $P$ be a faithful irreducible $(Q \lambda R)$ module over $\mathbb{F}_{p}$ and $G=P \lambda(Q \lambda R)$. Then $M=Q R$ is a maximal subgroup of $G$ and $M_{G}=1$. Suppose that $R$ is $S$-semiquasinormal in $G$. Then $R Q^{x}=Q^{x} R$ for any element $x \in G$. Since $r<q$ and $R$ is cyclic, $R \leq N_{G}\left(Q^{x}\right)$ for all $x \in G$ by [19, Chapter IV, Satz 2.8]. Then $M=Q R \leq N_{G}(Q)<G$. Since $M$ is maximal in $G, M=N_{G}(Q)$. Then $M^{x}=\left(N_{G}(Q)\right)^{x}=N_{G}\left(Q^{x}\right)$, and so $R \leq M^{x}$ for all $x \in G$. Hence $R \leq M_{G}=1$, a contradiction. Thus $R$ is not $S$-semiquasinormal in $G$. Finally, note that $Q^{G} \leq P Q$. Hence $R$ is $\tau$-quasinormal in $G$.

A group $G$ is called a $P S T$-group if $S$-quasinormality is a transitive relation in $G$, that is, if $H$ is an $S$-quasinormal subgroup of $K$ and $K$ is an $S$ quasinormal subgroup of $G$, then $H$ is $S$-quasinormal in $G$. Soluble PSTgroups have been studied in [1], [6], [7] and [11]. By a result of Kegel [22], an $S$-quasinormal subgroup of a group $G$ is subnormal. This is not the case for $S$-semiquasinormality and $\tau$-quasinormality since a Sylow 2-subgroup of $S_{3}$, the symmetric group of degree 3 , is $S$-semiquasinormal and $\tau$-quasinormal but not subnormal.

Applying Kegel's result (mentioned above), PST-groups are exactly those groups in which every subnormal subgroup is $S$-quasinormal. This class contains the class of all groups in which normality is transitive ( $T$ groups) and the class of all groups in which quasinormality is transitive (PT-groups). The last two classes have been widely studied in [10], [12], [17], [30] and [39]. The structure of soluble PST-groups was obtained by Agrawal in [1]. A group $G$ is called an $S B T$-group (see [24]) if $S$-semiquasinormality is a transitive relation in $G$. The structure of soluble $S B T$ groups was obtained in [24]. Note also that $S B T$-groups form a proper subclass of the class of $P S T$-groups.

We say that a group $G$ is a TQT-group if $\tau$-quasinormality is a transitive relation in $G$. Our purpose here is to establish a structure of soluble TQTgroups.

THEOREM 1.1. Let $P$ be a Sylow p-subgroup of a group $G, Q$ a Sylow $q$ subgroup of $G$ such that $p \neq q$. The following statements are equivalent: 
(1) $G$ is a soluble TQT-group.

(2) $G$ is a supersoluble group which has an abelian normal Hall subgroup of odd order $D$ such that $G / D$ is nilpotent, every subgroup of $D$ is normal in $G$, and every subgroup of $G$ is $\tau$-quasinormal in $G$. Moreover, if $p \notin \pi(D)$ and $q \notin \pi(D)$, then the order of $[P, Q]$ divides $r$, where $r \in \pi(D)$, and if $|[P, Q]|=r$, then $r>p, r>q$ and the Sylow r-subgroup of $G$ is cyclic.

The following example indicates that $S B T$-groups form a proper subclass of the class of $T Q T$-groups.

Example 2. Let $G=\left\langle x, y, z \mid x^{11}=y^{5}=z^{2}=[y, z]=1, x^{y}=x^{3}, x^{z}=x^{-1}\right\rangle$. Note that $\left\langle y^{x}\right\rangle$ is a Sylow5-subgroup of $G$ and $\langle z\rangle$ is a Sylow 2-subgroup of $G$, but $\left\langle y^{x}\right\rangle\langle z\rangle \neq\langle z\rangle\left\langle y^{x}\right\rangle$. By [24, Theorem 3.1], if $G$ is a soluble $S B T$-group, then every subgroup of $G$ is $S$-semiquasinormal in $G$. Thus $G$ is not an $S B T$-group. Finally, note that every subgroup of $G$ is $\tau$-quasinormal in $G$. Hence $G$ is a TQT-group.

The proof of Theorem 1.1 relies on the following result.

TheOREm 1.2. Let $G$ be a group. The following statements are equivalent:

(1) Every subgroup of $F^{*}(G)$ is $\tau$-quasinormal in $G$.

(2) $G$ is a supersoluble group and every subgroup of $F(G)$ is $\tau$-quasinormal in $G$.

(3) $G=D \lambda M$ is a supersoluble group, where $M$ is a nilpotent subgroup and $D$ is a Hall abelian subgroup of $G$ of odd order such that every subgroup of $D$ is normal in $G$.

(4) $G$ is a soluble group and every subnormal subgroup of $G$ is $S$ quasinormal in $G$.

In this theorem $F^{*}(G)$ denotes the generalized Fitting subgroup of $G$, that is, the product of all normal quasinilpotent subgroups of $G$; see [21, Chapter X].

Note that, in view of Theorem 1.2, the class of soluble groups in which every subnormal subgroup is $S$-quasinormal (PST-groups) coincides with the class of groups in which every subgroup of the generalized Fitting subgroup is $\tau$-quasinormal.

We shall prove Theorem 1.2 in Section 4. But before, in Section 3, we prove the following fact which is one of the main steps in the proof of Theorem 1.2. 
THEOREM 1.3. Let E be a normal subgroup of a group G. Suppose that for every non-cyclic Sylow p-subgroup $P$ of $F^{*}(E)$ there is a number $p^{k}$ such that $1<p^{k}<|P|$ and every subgroup of $P$ with order $p^{k}$ and every cyclic subgroup of $P$ with order 4 (if $p^{k}=2$ and $P$ is non-abelian) is $\tau$-quasinormal in $G$. Then each $G$-chief factor below $E$ is cyclic.

Note that Theorem 1.3 is independently interesting, as it not only generalizes the main results of many papers, but also considerably simplifies the proofs of some of them (see Section 5).

All unexplained notations and terminologies are standard. The reader is referred to [8] and [15] if necessary.

\section{Preliminaries.}

We will need to know a few facts about $\tau$-quasinormal subgroups.

Lemma 2.1 [28, Lemma 2.2]. Let $G$ be a group, $H \leq K \leq G$ and $L \leq G$.

(1) If $H$ is $\tau$-quasinormal in $G$, then $H$ is $\tau$-quasinormal in $K$.

(2) Suppose that $H$ is normal in $G$ and $\pi(K / H)=\pi(K)$. If $K$ is $\tau$-quasinormal in $G$, then $K / H$ is $\tau$-quasinormal in $G / H$.

(3) Suppose that $H$ is normal in $G$. Then $E H / H$ is $\tau$-quasinormal in $G / H$ for every $\tau$-quasinormal subgroup $E$ in $G$ satisfying $(|H|,|E|)=1$.

(4) If $H$ is $\tau$-quasinormal in $G$ and $H \leq O_{p}(G)$, then $H$ is $S$-quasinormal in $G$.

(5) Suppose that $H$ and $L$ are $\tau$-quasinormal in $G$. If $H L=L H$ and $\pi(H \cap L)=\pi(H)=\pi(L)$, then $H \cap L$ is $\tau$-quasinormal in $G$.

Lemma $2.2[28$, Lemma 2.5]. Let $N$ be an elementary abelian normal $p$ subgroup of a group $G$ for some prime $p$. Assume that there is a number $p^{k}$ such that $1<p^{k}<|N|$ and every subgroup of $N$ with order $p^{k}$ is $\tau$-quasinormal in $G$. Then some maximal subgroup of $N$ is normal in $G$.

Lemma 2.3 [27, Theorem 1.4]. Let $P$ be a Sylow p-subgroup of a group $G$, where $p$ is the smallest prime dividing $|G|$. Suppose that there is a number $p^{k}$ such that $1<p^{k}<|P|$ and every subgroup of $P$ with order $p^{k}$ and every cyclic subgroup of $P$ with order 4 (if $p^{k}=2$ and $P$ is non-abelian) is $\tau$ quasinormal in $G$. Then $G$ is p-nilpotent.

Lemma 2.4 [27, Lemmas 2.8 and 2.9]. Let $V$ be a $\tau$-quasinormal subgroup of order 4 of a group $G$. 
(1) If $V=A \times B$, where $|A|=|B|=2$ and $A$ is $\tau$-quasinormal in $G$, then $B$ is $\tau$-quasinormal in $G$.

(2) If $V=\langle x\rangle$ is cyclic, then $\left\langle x^{2}\right\rangle$ is $\tau$-quasinormal in $G$.

Recall that a formation $\mathfrak{\wp}$ is a class of groups which is closed under taking homomorphic images and such that each group $G$ has the smallest normal subgroup (denoted by $G^{\widetilde{r}}$ ) whose quotient is in $\widetilde{\wp}$. A formation $\widetilde{\wp}$ is

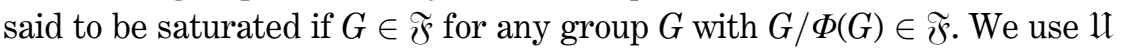
to denote the class of all supersoluble groups; $Z_{\mathfrak{l l}}(G)$ denotes the largest normal subgroup of a group $G$ whose $G$-chief factors are cyclic; see [15, p. 389]. The symbol $\mathfrak{H}(p-1)$ denotes the formation of all abelian groups of exponent dividing $p-1$; see [35].

LEMMA 2.5 [36, Lemma 2.2]. Let $E$ be a normal p-subgroup of a group $G$. If $E \leq Z_{\mathfrak{l} l}(G)$, then $\left(G / C_{G}(E)\right)^{\mathfrak{q}(p-1)} \leq O_{p}\left(G / C_{G}(E)\right)$.

Lemma 2.6 [4, Lemma 4]. Let $P$ be a p-subgroup of a group $G$, where $p>2$. Suppose that all subgroups of $P$ of order $p$ are $S$-quasinormal in $G$. If a is a $p^{\prime}$-element of $N_{G}(P) \backslash C_{G}(P)$, then a induces in $P$ a fixed-point free automorphism.

LEMMA 2.7 [36, Lemma 2.8]. Let $A, B, E$ be normal subgroups of a group $G$. Suppose that $G=A B$. If $E \leq Z_{\mathfrak{l l}}(A) \cap Z_{\mathfrak{l l}}(B)$ and $(|G: A|,|G: B|)=1$, then $E \leq Z_{\mathfrak{l l}}(G)$.

Lemma 2.8 [36, Theorem C]. Let $E$ be a normal subgroup of a group $G$. If every G-chief factor below $F^{*}(E)$ is cyclic, then every G-chief factor below $E$ is cyclic.

\section{Proof of Theorem 1.3.}

First we prove that if $W$ is a normal subgroup of $G$ and for every noncyclic Sylow $p$-subgroup $P$ of $W$ there is a number $p^{k}$ such that $1<p^{k}<|P|$ and every subgroup of $P$ with order $p^{k}$ and every cyclic subgroup of $P$ with order 4 (if $p^{k}=2$ and $P$ is non-abelian) is $\tau$-quasinormal in $G$, then each $G$ chief factor below $W$ is cyclic. Suppose that this is false and consider a counterexample $(G, W)$ for which $|G||W|$ is minimal. Let $P$ be a Sylow $p$ subgroup of $W$, where $p$ is the smallest prime dividing $|W|, C=C_{G}(P)$. If $P$ is not a non-abelian 2-group we use $\Omega$ to denote the subgroup $\Omega_{1}(P)$. Otherwise, $\Omega=\Omega_{2}(P)$. 
(1) If $Y$ is a Hall subgroup of $W$, the hypothesis is still true for $(Y, Y)$. If, in addition, $Y$ is normal in $G$, then the hypothesis also holds for $(G / Y, W / Y)$ and for $(G, Y)$.

Let $Y$ be a Hall subgroup of $W$ and $Y_{q}$ a non-cyclic Sylow $q$-subgroup of $Y$. Then $Y_{q}$ is a non-cyclic Sylow $q$-subgroup of $W$. By hypothesis and Lemma 2.1(1), there is a number $q^{k}$ such that $1<q^{k}<\left|Y_{q}\right|$ and every subgroup of $Y_{q}$ with order $q^{k}$ and every cyclic subgroup of $Y_{q}$ with order 4 (if $q^{k}=2$ and $Y_{q}$ is non-abelian) is $\tau$-quasinormal in $Y$. Thus the hypothesis is still true for $(Y, Y)$. Now let $Y$ be a Hall subgroup of $W$ which is normal in $G, Q Y / Y$ a non-cyclic Sylow $q$-subgroup of $W / Y$, where $q$ divides $|W / Y|$. Then $Q$ is a non-cyclic Sylow $q$-subgroup of $W$, so by hypothesis there is a number $q^{k}$ such that $1<q^{k}<|Q|$ and every subgroup of $Q$ with order $q^{k}$ and every cyclic subgroup of $Q$ with order 4 (if $q^{k}=2$ and $Q$ is non-abelian) is $\tau$ quasinormal in $G$. If $H^{*} / Y$ is a subgroup of $Q Y / Y$ with order $\left|H^{*} / Y\right|=q^{k}$, then $H^{*}=Y \lambda H$, where $H$ is a Sylow $q$-subgroup of $H^{*}$ and $H \leq Q$. Clearly, $|H|=q^{k}$, hence $H^{*} / Y=H Y / Y$ is $\tau$-quasinormal in $G / Y$ by Lemma 2.1(3). If $q^{k}=2, H^{*} / Y$ is a cyclic subgroup with order 4 and $Q Y / Y$ is non-abelian, then $Q$ is non-abelian and similarly $H^{*} / Y$ is $\tau$-quasinormal in $G / Y$. Thus the hypothesis is still holds for $(G / Y, W / Y)$. Hence the hypothesis is true for $(G, Y)$.

(2) If $Y$ is a non-identity normal Hall subgroup of $W$, then $Y=W$.

Since $Y$ is a characteristic subgroup of $W$, it is normal in $G$ and by (1) the hypothesis is still true for $(G / Y, W / Y)$ and for $(G, Y)$. If $Y \neq W$, the minimal choice of $(G, W)$ implies that $W / Y \leq Z_{\mathfrak{l}}(G / Y)$ and $Y \leq Z_{\mathfrak{l}}(G)$. Hence $W \leq Z_{\mathfrak{l}}(G)$, a contradiction.

(3) If $W \neq P$, then $W$ is not p-nilpotent.

Indeed, if $W$ is $p$-nilpotent, then by (2), $p$ does not divide $|W|$, contrary to the choice of $p$.

(4) $P$ is not cyclic.

Since $p$ is the smallest prime dividing $|W|$, it follows from (3) and [19, Chapter IV, Satz 2.8].

(5) $W=P$ is not a minimal normal subgroup of $G$.

Suppose that $W \neq P$. In view of (4), $P$ is not cyclic. Then by hypothesis and Lemma 2.1(1), there is a number $p^{k}$ such that $1<p^{k}<|P|$ and every subgroup of $P$ with order $p^{k}$ and every cyclic subgroup of $P$ with order 4 (if 
$p^{k}=2$ and $P$ is non-abelian) is $\tau$-quasinormal in $W$. Then Lemma 2.3 implies that $W$ is $p$-nilpotent, which contradicts (3). Therefore $W=P$. Suppose that $P$ is a minimal normal subgroup of $G$. Then by Lemma 2.2 some maximal subgroup of $P$ is normal in $G$, which contradicts the minimality of $P$.

(6) $|N| \leq p^{k}$ for any minimal normal subgroup $N$ of $G$ contained in $P$.

Assume that $p^{k}<|N|$. Then every subgroup of $N$ with order $p^{k}$ is $\tau$ quasinormal in $G$, so by Lemma 2.2 some maximal subgroup of $N$ is normal in $G$, a contradiction. Thus we have (6).

(7) If $P / N \leq Z_{\mathfrak{l}}(G / N)$ for every minimal normal subgroup $N$ of $G$ contained in $P$, then $\Phi(P) \neq 1$.

Suppose that $\Phi(P)=1$. Then $P$ is an elementary abelian $p$-group. Let $R$ be any minimal normal subgroup of $G$ contained in $P$ such that $R \neq N$. Then $P / R \leq Z_{\mathfrak{l l}}(G / R)$. Thus from the $G$-isomorphism $N \simeq N R / R$ we deduce that $N \leq Z_{\mathfrak{l}}(G)$, hence $P \leq Z_{\mathfrak{l}}(G)$. This contradiction shows that $N$ is the only minimal normal subgroup of $G$ contained in $P$. Now let $N_{1}$ be any maximal subgroup of $N$. In view of (6), $\left|N_{1}\right|<p^{k}$. We claim that $N_{1}$ is $\tau$ quasinormal in $G$. Let $S$ be a complement of $N$ in $P, B$ a subgroup of $S$ such that $\left|N_{1}\right||B|=p^{k}$. Then by hypothesis $N_{1} B$ is $\tau$-quasinormal in $G$. Hence $N_{1} B \cap N=N_{1}(B \cap N)=N_{1}$ is $\tau$-quasinormal in $G$ by Lemma 2.1(5). Therefore every maximal subgroup of $N$ is $\tau$-quasinormal in $G$. Hence some maximal subgroup of $N$ is normal in $G$ by Lemma 2.2, a contradiction. Therefore $\Phi(P) \neq 1$.

(8) $C_{G}(P / \Phi(P)) / C$ is a p-group.

Suppose that this is false. Let $a \notin C$ be a $p^{\prime}$-element of $C_{G}(P / \Phi(P))$ and $G_{0}=P \lambda(G / C)$. Then $a C$ is a non-identity $p^{\prime}$-element of $G / C$ and $a C \in C_{G_{0}}(P / \Phi(P))$, which contradicts [18, Chapter 5, Theorem 1.4]. Thus $C_{G}(P / \Phi(P)) / C$ is a $p$-group.

(9) $P / \Phi(P) \not \leq Z_{\mathfrak{l l}}(G / \Phi(P))$.

Suppose that $P / \Phi(P) \leq Z_{\mathfrak{l}}(G / \Phi(P))$. Then Lemma 2.5 implies that $\left(G / C_{G}(P / \Phi(P))\right)^{\mathfrak{2}(p-1)}$ is a $p$-group. Hence, in view of $(8),(G / C)^{\mathfrak{I}(p-1)}$ is a $p$-group. Let $H / K$ be any chief factor of $G$ below $\Phi(P)$. Then $G / C_{G}(H / K) \in \mathfrak{U}(p-1)$, since $O_{p}\left(G / C_{G}(H / K)\right)=1$ by [34, Chapter 1, Lemma 3.9]. Hence $|H / K|=p$ in view of [34, Chapter 1, Lemma 4.1]. Thus $W=P \leq Z_{\mathfrak{l l}}(G)$. This contradiction completes the proof of (9).

(10) $|P|>p^{k+1}$. 
Suppose that $|P|=p^{k+1}$. Then by hypothesis every maximal subgroup of $P$ is $\tau$-quasinormal in G. By (6) and Lemma 2.1(2) the hypothesis is still true for $(G / N, P / N)$ for any minimal normal subgroup $N$ of $G$ contained in $P$, so $P / N \leq Z_{\mathfrak{l l}}(G / N)$ by the choice of $(G, W)=(G, P)$, whence, in view of (7), $\Phi(P) \neq 1$. Then $P / \Phi(P) \leq Z_{\mathfrak{l}}(G / \Phi(P))$, which contradicts (9).

(11) $k>1$.

Suppose that $k=1$.

(a) $G$ has a normal subgroup $1 \neq R \leq P$ such that $P / R$ is a non-cyclic chieffactor of $G, R \leq Z_{\mathfrak{l l}}(G)$ and $V \leq R$ for any normal subgroup $V \neq P$ of $G$ contained in $P$.

Let $P / R$ be a chief factor of $G$. Then $R \neq 1$ by (5). Moreover, the hypothesis holds for $(G, R)$, so $R \leq Z_{\mathfrak{l}}(G)$ and $P / R$ is not cyclic by the choice of $(G, P)=(G, W)$. Now let $V$ be any normal subgroup of $G$ with $V<P$. Then $V \leq Z_{\mathfrak{l}}(G)$. If $V \not \leq R$, then from the $G$-isomorphism $P / R=$ $V R / R \simeq V / V \cap R$ we deduce that $P \leq Z_{\mathfrak{l l}}(G)$, contrary to the choice of $(G, P)$. Hence $V \leq R$.

(b) $C_{G}(\Omega) / C$ is a p-group.

Let $G_{0}=P \lambda(G / C)$. If $a$ is a $p^{\prime}$-element of $C_{G}(\Omega)$ such that $a \notin C$, then $a C$ is a non-identity $p^{\prime}$-element of $G / C$ and $a C \in C_{G_{0}}(\Omega)$, which contradicts [16, Theorem 2.4]. Thus $C_{G}(\Omega) / C$ is a $p$-group.

(c) $\Omega \not \leq Z_{\mathfrak{l l}}(G)$.

Suppose that $\Omega \leq Z_{\mathfrak{l}}(G)$. Then by Lemma $2.5,\left(G / C_{G}(\Omega)\right)^{\mathfrak{A}(p-1)}$ is a $p$-group. Hence, in view of (b), $(G / C)^{\mathfrak{I}(p-1)}$ is a $p$-group. Therefore $G / C_{G}(P / R) \in \mathfrak{U}(p-1)$, which implies that $|P / R|=p$. Hence we have (c).

(d) $\Omega=P$.

Indeed, if $\Omega<P$, then, in view of (a), $\Omega \leq Z_{\mathfrak{l}}(G)$, which contradicts (c).

(e) $P \leq O^{p}(G)$.

Suppose that $P \nsubseteq O^{p}(G)$. Then from the $G$-isomorphism $P O^{p}(G) / O^{p}(G) \simeq$ $P /\left(O^{p}(G) \cap P\right)$ we deduce that $G$ has a cyclic chief factor $P / V$, where $O^{p}(G) \cap P \leq V$, which contradicts (a).

(f) There is a prime $q \neq p$ such that $q$ divides $|G: C|$.

Otherwise, any $G_{p}$-chief factor of $P$, where $G_{p}$ is a Sylow $p$-subgroup of $G$, is a chief factor of $G$, which implies $W=P \leq Z_{\mathfrak{l l}}(G)$, a contradiction. 


\section{Final contradiction for (11).}

In view of (d) we have $P / R=\left(V_{1} R / R\right)\left(V_{2} R / R\right) \ldots\left(V_{t} R / R\right)$, where $V_{i}$ is a cyclic group of order $p$ or order 4 and $V_{i} R / R$ is a cyclic group of order $p$. By hypothesis, $V_{i}$ is $\tau$-quasinormal in $G$. In view of Lemma 2.1(4), $V_{i}$ is $S$-quasinormal in $G$, so if $Q$ is a Sylow $p^{\prime}$-subgroup of $G$, then $V_{i} Q=Q V_{i}$. Hence $P \cap V_{i} Q=V_{i}(P \cap Q)=V_{i} \unlhd V_{i} Q$. Suppose that $p=2$. Then in view of [19, Chapter IV, Satz 2.8], $V_{i} Q$ is nilpotent, so $Q \leq C_{G}\left(V_{i}\right)$. Therefore $O^{p}(G) \leq C_{G}(P / R)$, whence $C_{G}(P / R)=G$, which contradicts (a). Thus $p>2$. Notice also that $O^{p}(G) \neq G$. Indeed, if $O^{p}(G)=G$, then, in view of [31, Lemma A], $V_{i} R / R$ is normal in $G / R$, so $V_{i} R / R=P / R$ is cyclic, which contradicts (a). Thus $O^{p}(G) \neq G$. Now we claim that there is a prime $q \neq p$ such that $O^{q}(G) \neq G$. Indeed, assume that $O^{q}(G)=G$ for all primes $q \neq p$. Then for every $G$-chief factor $H / K$ of order $p$ we have $C_{G}(H / K)=G$. In particular, for a minimal normal subgroup $L$ of $G$ contained in $R$ we have $L \leq Z(G)$. Hence $C_{P}(a) \neq 1$ for any $p^{\prime}$-element $a \in G \backslash C$. Since every subgroup $V_{i}$ is $S$-quasinormal in $G$, in view of Lemma 2.6, $O^{p}(G) \leq C$, which contradicts (f). Therefore there is a prime $q \neq p$ such that $P \leq O^{q}(G) \neq G$. Since $P \leq O^{p}(G) \neq G$ by (e), we have that $P \leq Z_{\mathfrak{l l}}(G)$ by Lemma 2.7 , a contradiction.

Hence $k>1$.

(12) If $P$ is a non-abelian 2-group, then $p^{k}>4$.

Since $P$ is a non-abelian 2-group, it has a cyclic subgroup $H=\langle x\rangle$ with $|H|=4$. Suppose that $p^{k}=4$. Then by hypothesis $H$ is $\tau$-quasinormal in $G$. Therefore $\left\langle x^{2}\right\rangle$ is $\tau$-quasinormal in $G$ by Lemma 2.4(2). Now note that if $G$ has a subgroup $V=A \times B$ of order 4 such that $|A|=2$ and $A$ is $\tau$-quasinormal in $G$, then $V$ and $B$ are $\tau$-quasinormal in $G$ (we use here some arguments of the proof of Theorem 1 in [32]). Indeed, $V$ is $\tau$-quasinormal in $G$ by hypothesis. Hence $B$ is $\tau$-quasinormal in $G$ by Lemma 2.4(1). Therefore some subgroup $Z$ of $Z(P)$ with $|Z|=2$ is $\tau$-quasinormal in $G$, so every subgroup of $P$ with order 2 is $\tau$-quasinormal in $G$ by Lemma 2.4(1), which contradicts (11).

(13) If $N$ is an abelian minimal normal subgroup of $G$ contained in $P$, then the hypothesis is still holds for $(G / N, P / N)$.

In view of (10), $|P|>p^{k+1}$. If either $p>2$ and $|N|<p^{k}$ or $p=2$ and $|N|<p^{k-1}$, it is clear. So let either $p>2$ and $|N|=p^{k}$ or $p=2$ and $|N| \in\left\{p^{k}, p^{k-1}\right\}$. In view of (11), $k>1$. Then by hypothesis every subgroup of $P$ with order $p^{k}$ is $\tau$-quasinormal in $G$. Suppose that $|N|=p^{k}$. Then $N$ is 
non-cyclic and hence every subgroup of $G$ containing $N$ is non-cyclic. Let $N \leq K \leq P$, where $|K: N|=p$. Since $K$ is non-cyclic, it has a maximal subgroup $L \neq N$. Then $K=L N$ is $\tau$-quasinormal in $G$, as it is the product of two $\tau$-quasinormal in $G$-subgroups. Thus if either $p>2$ or $P / N$ is abelian, the hypothesis is true for $(G / N, P / N)$ by Lemma 2.1(2). Next suppose that $P / N$ is a non-abelian 2-group. Then $P$ is non-abelian, so $p^{k}>4$ by (12). Let $N \leq K \leq V$, where $|V: N|=4$ and $|V: K|=2$. Let $K_{1}$ be a maximal subgroup of $V$ such that $V=K_{1} K$. Suppose that $K_{1}$ is cyclic. Then $N \nsubseteq K_{1}$, so $V=K_{1} N$, which implies $|N|=4$. But then $p^{k}=4$, which contradicts (12). Hence $K_{1}$ is non-cyclic and as above one can show that $K_{1}$ is $\tau$-quasinormal in $G$. Therefore every cyclic subgroup of $P / N$ with order 2 and order 4 is $\tau$-quasinormal in $G / N$ by Lemma 2.1(2). Finally, suppose that $|N|=p^{k-1}$. If $|N|>2$, then as above one can show that every subgroup of $P / N$ with order 2 and order 4 (if $P / N$ is non-abelian) is $\tau$-quasinormal in $G / N$. Now, suppose that $|N|=2$ and $P / N$ is non-abelian. Then $P$ is non-abelian and $p^{k}=4$, which contradicts (12). Hence we have (13).

\section{Final contradiction.}

In view of (7) and (13), $\Phi(P) \neq 1$, whence $P / \Phi(P) \leq Z_{\mathfrak{l l}}(G / \Phi(P))$ by (13), which contradicts (9). Therefore each $G$-chief factor below $W$ is cyclic.

Since $F^{*}(E)$ is characteristic in $E$, it is normal in $G$. Hence taking $W=F^{*}(E)$ we deduce from above that each $G$-chief factor below $F^{*}(E)$ is cyclic. Then each $G$-chief factor below $E$ is cyclic by Lemma 2.8. This completes the proof of this theorem.

\section{Proof of Theorems 1.1 and 1.2 .}

The following lemma is well known.

Lemma 4.1. Let $G$ be a group and $N \unlhd G$. Then $(G / N)^{\Re}=G^{\Re} N / N$.

Lemma 4.2. Let $G$ be a metanilpotent group. If every subgroup of $G^{\Re}$ of prime power order is $\tau$-quasinormal in $G$, then $G^{\Re}$ is a Hall subgroup of $G$.

Proof. Suppose that this lemma is false and let $G$ be a counterexample with minimal $|G|$. Then $D=G^{\Re} \neq 1$. Let $N$ be a minimal normal subgroup of $G$. Then by Lemma 4.1, $(G / N)^{\Re}=G^{\Re} N / N=D N / N$. Let $V / N$ be any subgroup of $D N / N$ of prime power order $p^{a}$. Then $V=(V \cap D) N$. If $N$ is a 
$p$-group, then $V$ is a $p$-group and so it is $\tau$-quasinormal in $G$ by hypothesis. Then $V / N$ is $\tau$-quasinormal in $G / N$ by Lemma 2.1(2). Otherwise, $V=N \lambda V_{p}$, where $V_{p}$ is a Sylow $p$-subgroup of $V$. By hypothesis, $V_{p}$ is $\tau$ quasinormal in $G$, so $V / N$ is $\tau$-quasinormal in $G / N$ by Lemma 2.1(3). Therefore the hypothesis holds for $G / N$, so $(G / N)^{\Re}=D N / N$ is a Hall subgroup of $G / N$ by the choice of $G$. Now assume that $G$ has two minimal normal subgroups $H$ and $R$ such that $H$ is a $p$-group and $R$ is a $q$-group, where $p \neq q$. Then, without loss of generality, we may let $H \leq D$. Then $D R / R$ is a Hall subgroup of $G / R$ by the choice of $G$. Let $D_{p}$ be a Sylow $p$ subgroup of $D$. Then $D_{p} R / R$ is a Sylow $p$-subgroup of $D R / R$, and so it is a Sylow $p$-subgroup of $G / R$. Hence $D_{p}$ is a Sylow $p$-subgroup of $G$. Suppose that $D_{p} \neq D$ and let $D_{r}$ be a Sylow $r$-subgroup of $D$, where $r \neq p$. Then we see as above that $D_{r}$ is a Sylow $r$-subgroup of $G$. Thus $D$ is a Hall subgroup of $G$. Now we consider the case when all minimal normal subgroups of $G$ are $p$ groups. Then $F(G)=O_{p}(G)$ is a Sylow $p$-subgroup of $G$ and so $D \leq O_{p}(G)$. If $H \neq D$, then, by using the same argument as above, we see that $D$ is a Sylow $p$-subgroup of $G$. Hence, we may put $H=D$. Now we claim that $\Phi=\Phi\left(O_{p}(G)\right)=1$. In fact, if we assume that $\Phi \neq 1$, then $D \Phi / \Phi=$ $G^{\Re} \Phi / \Phi=(G / \Phi)^{\Re}$ is a Hall subgroup of $G / \Phi$. If $H \leq \Phi$, then $G / \Phi$ is a nilpotent group. But $O_{p}(G) \unlhd G$, and so $\Phi \leq \Phi(G)$. This shows that $G$ is nilpotent so that $H=G^{\Re}=1$, a contradiction. Hence $H \nsubseteq \Phi$, and so $H \Phi / \Phi$ is a non-identity $p$-group. Consequently, we have $H \Phi=O_{p}(G)$. It follows that $H=O_{p}(G)$. This contradiction shows that $\Phi\left(O_{p}(G)\right)=1$. Hence $O_{p}(G)$ is an elementary abelian group. Hence every subgroup of $O_{p}(G)$ is normal in $G$. Let $O_{p}(G)=\langle a\rangle \times\left\langle a_{2}\right\rangle \times \ldots \times\left\langle a_{t}\right\rangle$, where $\left\langle a_{i}\right\rangle$ is a minimal normal subgroup of $G$, and $\langle a\rangle=H$. Write $a_{1}=a a_{2} \ldots a_{t}$. Then since $\left\langle a_{1}\right\rangle \cap\left\langle a_{2}\right\rangle \cap \ldots \cap\left\langle a_{t}\right\rangle=1$, we have $O_{p}(G)=\left\langle a_{1}\right\rangle \times\left\langle a_{2}\right\rangle \times \ldots \times\left\langle a_{t}\right\rangle$. Since $G$ is not nilpotent, $O_{p}(G) \nsubseteq Z(G)$. Hence there is an index $i$ such that $a_{i} \notin Z(G)$. It is clear that $\left\langle a_{i}\right\rangle \unlhd G$ and that $\left\langle a_{i}\right\rangle \neq H$. Since $H=D=G^{\Re},\left\langle a_{i}\right\rangle H / H \leq$ $Z(G / H)$. Hence from the $G$-isomorphism $\left\langle a_{i}\right\rangle H / H \simeq\left\langle a_{i}\right\rangle$ we have $\left\langle a_{i}\right\rangle \leq Z(G)$, a contradiction. The lemma is proved.

Proof of Theorem 1.2. If every subgroup of $F^{*}(G)$ is $\tau$-quasinormal in $G$, then $G$ is supersoluble by Theorem 1.3. Hence, in view of [21, Chapter X], every subgroup of $F(G)=F^{*}(G)$ is $\tau$-quasinormal in $G$. So (1) implies (2).

We now show that (2) implies (3). Suppose that $G$ is a supersoluble group and every subgroup of $F(G)$ is $\tau$-quasinormal in $G$. Then $D=G^{\Re}$ is a Hall subgroup of $G$ by Lemma 4.2. Since every chief factor of $G$ is cyclic, it follows that $D$ is odd order. By using well known Schur-Zassenhaus's 
theorem, we see that $D$ has a complement $M$ in $G$. Since $G / D \simeq M$ is nilpotent, $M$ is a Hall nilpotent subgroup of $G$ and $G=D \lambda M$. Now let $H$ be an arbitrary subgroup of $D, \pi(H)=\left\{p_{1}, p_{2}, \ldots, p_{t}\right\}$ and $P_{i}$ be a Sylow $p_{i^{-}}$ subgroup of $H$. Since $D$ is a normal nilpotent subgroup of $G, P_{i}$ is subnormal in $G$. By hypothesis, $P_{i}$ is $\tau$-quasinormal in $G$. Moreover, $P_{i} \leq O_{p_{i}}(G)$, so $P_{i}$ is $S$-quasinormal in $G$ by Lemma 2.1(4). Then $O^{p_{i}}(G) \leq N_{G}\left(P_{i}\right)$ by [31, Lemma A]. Since $D=G^{\Re} \leq O^{p_{i}}(G)$ and $D$ is a Hall subgroup of $G$, we have that $O^{p_{i}}(G)=G$ for all $i \in\{1,2, \ldots, t\}$. Hence $P_{i}$ is normal in $G$, so $H=P_{1} P_{2} \ldots P_{t}$ is normal in $G$. Finally, since $D$ is odd order, it follows that $D$ is abelian. Therefore, (3) is a consequence of (2).

Now we show that (3) implies (4). Assume that $G=D \lambda M$ is a supersoluble group, where $M$ is a nilpotent subgroup and $D$ is a Hall abelian subgroup of $G$ of odd order such that every subgroup of $D$ is normal in $G$. Let $H$ be a subnormal subgroup of $G$ and $P$ a Sylow $p$-subgroup of $G$. If $p \in \pi(D)$, then $P \leq D$, so $P$ is normal in $G$ by hypothesis. Thus $H P=P H$. Assume that $p \notin \pi(D)$. By using well known Schur-Zassenhaus's theorem, we see that $D$ has a complement $L$ in $G$ such that $P \leq L$. Then $P$ is a Sylow $p$-subgroup of $L$. Since $H$ is subnormal in $G$ and $(|D|,|L|)=1$, we have that $H=(H \cap D)(H \cap L)$. By hypothesis, $H \cap D$ is normal in $G$. Moreover, $G / D \simeq L$ is nilpotent. Hence $P$ is normal in $L$. Then $(H \cap L) P=P(H \cap L)$. Thus $H P=(H \cap D)(H \cap L) P=P H$. Therefore, (4) is a consequence of (3).

Finally, we show that (4) implies (1). Suppose that $G$ is a soluble group and every subnormal subgroup of $G$ is $S$-quasinormal in $G$. Then $F(G)=F^{*}(G)$ by [21, Chapter X], so every subgroup of $F^{*}(G)$ is subnormal in $G$. Hence by hypothesis every subgroup of $F^{*}(G)$ is $S$-quasinormal in $G$, and, obviously, it is $\tau$-quasinormal in $G$. This completes the proof.

Proof of Theorem 1.1. First we show that (1) implies (2). Let $G$ be a soluble TQT-group. Then, in view of [21, Chapter X], $F^{*}(G)=F(G)$. Hence every Sylow subgroup of $F^{*}(G)$ is normal in $F^{*}(G)$, so every subgroup of $F^{*}(G)$ is $\tau$-quasinormal in $F^{*}(G)$. Moreover, $F^{*}(G)$ is $\tau$-quasinormal in $G$. Since $G$ is a $T Q T$-group, we have that every subgroup of $F^{*}(G)$ is $\tau$-quasinormal in $G$. By Theorem 1.2, $G$ has an abelian normal Hall subgroup $D$ such that $G / D$ is nilpotent and every subgroup of $D$ is normal in $G$. Let $H$ be a subgroup of $G$ and $S_{1}$ any Sylow subgroup of $D H$ such that $\left(|H|,\left|S_{1}\right|\right)=1$. Then $S_{1} \leq D$, so $H S_{1}=S_{1} H$. Hence $H$ is $\tau$-quasinormal in $D H$. Now let $S_{2}$ be any Sylow subgroup of $G$ such that $\left(|D H|,\left|S_{2}\right|\right)=1$. Then $S_{2} D / D$ is a Sylow subgroup of $G / D$. Since $G / D$ is nilpotent, $S_{2} D$ is normal in $G$. Then $(D H) S_{2}=H S_{2} D=S_{2}(D H)$. Hence $D H$ is $\tau$-quasinormal in $G$. Since $G$ is a TQT-group, $H$ is $\tau$-quasinormal in $G$. 
Now let $P$ be any Sylow $p$-subgroup of $G, Q$ a Sylow $q$-subgroup of $G$ such that $p \neq q, p \notin \pi(D)$ and $q \notin \pi(D)$. Then $P \cap D=1=Q \cap D$. Since $G / D$ is nilpotent, $D P$ and $D Q$ are normal in $G$. If $P$ is normal in $G$, then, in view of [19, Chapter III, Hilfssatz 1.6(c)(d)], $[P, Q] \leq[P, D Q] \leq P \cap D Q=1$. If $Q$ is normal in $G$, then similarly we have that $[P, Q]=1$. Suppose that $P<P^{G}$ and $Q<Q^{G}$. Let $r$ be a prime dividing $\left|D \cap P^{G} Q^{G}\right|$ and $C_{r}$ a subgroup of $G$ with $\left|G_{r}\right|=r$. Then $G_{r} \leq D$, so $C_{r}$ is normal in $G$. We may assume without loss that $r$ divides $\left|P^{G}\right|$. Since every subgroup of $G$ is $\tau$-quasinormal, $\left(\left|C_{r} Q\right|, p\right)=1$ and $\left(\left|C_{r} Q\right|,\left|P^{G}\right|\right) \neq 1$, we have that $C_{r} Q P$ is a subgroup of $G$. First assume that $C_{r} \leq P^{G} \cap Q^{G}$. Then $P^{G} \cap C_{r} Q P=C_{r} P$ and $Q^{G} \cap C_{r} Q P=C_{r} Q$, so $C_{r} Q \leq$ $N_{G}\left(C_{r} P\right)$ and $C_{r} P \leq N_{G}\left(C_{r} Q\right)$. Hence $[P, Q] \leq\left[C_{r} P, C_{r} Q\right] \leq C_{r} P \cap C_{r} Q=C_{r}$ by [19, Chapter III, Hilfssatz 1.6(c)(d)]. Now assume that $C_{r} \leq P^{G}$ and $C_{r} \nsubseteq Q^{G}$. Then $Q \leq N_{G}\left(C_{r} P\right)$ and $Q^{G} \cap C_{r} Q P=Q$, so $C_{r} P \leq N_{G}(Q)$. Hence $[P, Q] \leq\left[C_{r} P, Q\right] \leq C_{r} P \cap Q=1$ by [19, Chapter III, Hilfssatz 1.6(c)(d)]. If $C_{r} \nsubseteq P^{G}$ and $C_{r} \leq Q^{G}$, then similarly we have that $[P, Q]=1$. Finally, assume that $C_{r} \nsubseteq P^{G} Q^{G}$. Then $P^{G} \cap C_{r} Q P=P$ and $Q^{G} \cap C_{r} Q P=Q$, so $Q \leq N_{G}(P)$ and $P \leq N_{G}(Q)$. Hence $[P, Q] \leq P \cap Q=1$ by [19, Chapter III, Hilfssatz 1.6(d)]. Thus if $G$ contains a subgroup $C_{s}$ with $\left|C_{s}\right|=s$ such that $C_{s} \neq C_{r}$ and $s \in \pi\left(D \cap P^{G} Q^{G}\right)$, then similarly $[P, Q] \leq C_{s}$. Hence $[P, Q] \leq$ $C_{r} \cap C_{s}=1$. Now suppose that $|[P, Q]|=r$. Then $G$ has only one subgroup with order $r$. Hence the Sylow $r$-subgroup of $G$ is cyclic by [19, Chapter I, Satz 2.20]. Therefore, (2) is a consequence of (1).

$(2) \Rightarrow(1)$ It is evident.

\section{Some applications of Theorem 1.3.}

Buckley [13] obtained a description of nilpotent groups of odd order all of whose subgroups of prime order are normal. As a consequence, he also proved that a group of odd order is supersoluble if all its subgroups of prime order are normal. Applying the description of minimal nonsupersoluble groups due to Doerk [14] and Huppert [20], we can go further and prove that a group is supersoluble if all cyclic subgroups of prime order and order 4 are normal. Later, Srinivasan [37] proved that a group $G$ is supersoluble if every maximal subgroup of every Sylow subgroup of $G$ is normal in $G$. These results have been developed in various directions, especially in the framework of formation theory. In particular, if $\mathfrak{\mho}$ is a saturated formation containing all supersoluble groups and $G$ is a group with a normal subgroup $E$, then the following results are true. 
(1) If $G / E \in \widetilde{r}$ and the cyclic subgroups of $E$ of prime order and order 4 are either all $S$-quasinormal (Ballester-Bolinches and Pedraza-Aguilera [9], Asaad and Csörgö [4]) or all $S$-semiquasinormal (Li [23]) in $G$, then $G \in \mathfrak{r}$.

(2) If $G / E \in \mathfrak{\wp}$ and the cyclic subgroups of $F^{*}(E)$ of prime order and order 4 are either all $S$-quasinormal (Li and Wang [26]) or all $S$-semiquasinormal (L. Wang and Y. Wang [38]) in $G$, then $G \in \widetilde{F}$.

(3) If $G / E \in \mathfrak{\wp}$ and the maximal subgroups of every Sylow subgroup of $E$ are either all $S$-quasinormal (Asaad [2]) or all $S$-semiquasinormal ( $\mathrm{Li}$ [23]) in $G$, then $G \in \widetilde{\mho}$.

(4) If $G / E \in \widetilde{\Im}$ and the maximal subgroups of every Sylow subgroup of $F^{*}(E)$ are either all $S$-quasinormal (Li, Wang and Wei [25]) or all $S$-semiquasinormal (Wang [38]) in $G$, then $G \in \widetilde{F}$.

Theorem 1.3 not only generalizes all the results [2], [4], [9], [13], [23]-[26], [37], [38] mentioned above and some main results in [3], [5], [29], [33] but also gives shorter proofs of some of them.

Acknowledgments. The authors would like to thank the referee for his or her helpful comments and suggestions which have improved the original manuscript to its present form.

\section{REFERENCES}

[1] R. K. AGRAwaL, Finite groups whose subnormal subgroups permute with all Sylow subgroups, Proc. Amer. Math. Soc. 47 (1) (1975), pp. 77-83.

[2] M. AsAAD, On maximal subgroups of Sylow subgroups of finite groups, Comm. Algebra, 26 (1998), pp. 3647-3652.

[3] M. AsAAD, On the solvability of finite groups, Arch. Math., 51 (1988), pp. 289293.

[4] M. AsAAD - P. CsöRGö, The influence of minimal subgroups on the structure of finite groups, Arch. Math., 72 (1999), pp. 401-404.

[5] M. ASAAD - M. RAMADAN - A. SHAALAN, Influence of $\pi$-quasinormality on maximal subgroups of Sylow subgroups of Fitting subgroup of a finite group, Arch. Math., 56 (1991), pp. 521-527.

[6] A. Ballester-Bolinches - R. Esteban-Romero, Sylow permutable subnormal subgroups of finite groups, J. Algebra, 251 (2002), pp. 727-738.

[7] A. Ballester-Bolinches - R. Esteban-Romero, Sylow permutable subnormal subgroups of finite groups, II, Bull. Austral. Math. Soc., 64 (2001), pp. $479-486$

[8] A. Ballester-Bolinches - L. M. EzQuerro, Classes of Finite Groups, Springer, Dordrecht, 2006.

[9] A. Ballester-Bolinches - M. C. Pedraza-Aguilera, On minimal subgroups of finite groups, Acta Math. Hungar., 73 (1996), pp. 335-342. 
[10] J. C. Beidleman - B. Brewster - D. J. S. Robinson, Criteria for permutability to be transitive in finite groups, J. Algebra, 222 (1999), pp. 400-412.

[11] J. C. Beidleman - H. Heineken - M. F. Ragland, Solvable PST-groups, strong Sylow bases and mutually permutable products, J. Algebra, 321 (7) (2009), pp. 2022-2027.

[12] R. A. BRYCE - J. Cossey, The Wielandt subgroup of a finite soluble group, J. London Math. Soc., 40 (1989), pp. 244-256.

[13] J. BuCKLEY, Finite groups whose minimal subgroups are normal, Math. Z., 15 (1970), pp. 15-17.

[14] K. DoenK, Minimal nicht uberauflosbare, endlicher Gruppen, Math. Z., 91 (1966), pp. 198-205.

[15] K. Doerk - T. HAwkes, Finite Soluble Groups, Walter de Gruyter, Berlin New York, 1992.

[16] T. M. GaGen, Topics in Finite Groups, Cambridge University Press, 1976.

[17] W. GASCHÜTZ, Gruppen in denen das Normalteilersein transitiv ist, J. Reine Angew. Math., 198 (1957), pp. 87-92.

[18] D. Gorenstein, Finite Groups, Harper Row Publishers, New York Evanston - London, 1968.

[19] B. Huppert, Endliche Gruppen I, Springer, Berlin - Heidelberg - New York, 1967.

[20] B. HuPpert, Normalteiler and maximale Untergruppen endlicher Gruppen, Math. Z., 60 (1954), pp. 409-434.

[21] B. Huppert - N. Blackburn, Finite Groups III, Springer - Verlag, Berlin New York, 1982.

[22] O. H. KEGEL, Sylow-Gruppen and Subnormalteiler endlicher Gruppen, Math. Z., 78 (1962), pp. 205-221.

[23] Y. LI, On S-semipermutable and c-normal subgroups of finite groups, Arabian J. Sci. Engineering, 34(2A) (2009), pp. 167-175.

[24] Y. LI - L. WANG - Y. WANG, Finite groups in which (S)-semipermutability is a transitive relation, Internat. J. Algebra, 2(3) (2008), pp. 143-152.

[25] Y. Li - Y. WANG - H. WEI, The influence of $\pi$-quasinormality of some subgroups of a finite group, Arch. Math., 81 (2003), pp. 245-252.

[26] Y. LI - Y. WANG, The influence of minimal subgroups on the structure of a finite group, Proc. Amer. Math. Soc., 131 (2002), pp. 337-341.

[27] V. O. LukYANEnko - A. N. Skiba, On $\tau$-quasinormal and weakly $\tau$-quasinormal subgroups of finite groups, Math. Sci. Res. J., 12(10) (2008), pp. 243-257.

[28] V. O. Lukyanenko - A. N. SkiBa, On weakly $\tau$-quasinormal subgroups of finite groups, Acta Math. Hungar., 125 (3) (2009), pp. 237-248.

[29] M. RAMADAN, Influence of normality on maximal subgroups of Sylow subgroups of a finite group, Acta Math. Hungar., 59 (1992), pp. 107-110.

[30] D. J. S. RoBinson, A note of finite groups in which normality is transitive, Proc. Amer. Math. Soc., 19 (1968), pp. 933-937.

[31] P. ScHмID, Subgroups permutable with all Sylow subgroups, J. Algebra, 182 (1998), pp. 285-293.

[32] V. I. Sergienko, A criterion for the p-solubility of finite groups, Mat. Zam., 9 (1971), pp. 375-383 (Russian, English translation in Math. Notes, 9 (1971), pp. 216-220). 
[33] A. SHaAlan, The influence of S-permutability of some subgroups, Acta Math. Hungar., 56 (1990), pp. 287-293.

[34] L. A. Shemetkov, Formations of Finite Groups, Nauka, Moscow, 1978.

[35] L. A. Shemetkov - A. N. SkibA, Formations of Algebraic Systems, Nauka, Moscow, 1989.

[36] A. N. SKIBA, A characterization of the hypercyclically embedded subgroups of finite groups, J. Pure Appl. Algebra, (2010), DOI: 10.1016/j.jpaa.2010.04.017.

[37] S. SRINIVASAN, Two sufficient conditions for supersolubility of finite groups, Israel J. Math., 3 (35) (1980), pp. 210-214.

[38] L. WANG - Y. WANG, On S-semipermutable maximal and minimal subgroups of Sylow p-subgroups of finite groups, Comm. Algebra, 34 (2006), pp. 143-149.

[39] G. ZACHER, I gruppi risolubili finiti in cui $i$ sottogrupi di composizione coincidono con $i$ sottogruppi quasi-normali, Atti Accad. Naz. Lincei Rend. Cl. Sci. Fis. Mat. Natur., 37 (8) (1964), pp. 150-154.

Manoscritto pervenuto in redazione il 20 luglio 2010. 